\title{
Techniques for noninvasive molecular imaging of atherosclerotic plaque
}

Jason M. Tarkin and James H. F. Rudd

We thank Federico Caobelli and Frank M. Bengel for their Correspondence (In vivo evaluation of atherosclerotic plaques and culprit lesions using noninvasive techniques. Nat. Rev. Cardiol. doi:10.1038/ nrcardio.2014.80-c1) $)^{1}$ on our Review (PET imaging of inflammation in atherosclerosis. Nat. Rev. Cardiol. 11, 443-457; 2014) $)^{2}$ and welcome their comments. We agree that advances in molecular imaging of plaques have the potential to change how we think about the management of atherosclerosis, and help to direct treatment towards patients at the highest risk of future clinical events. Several noninvasive molecular imaging platforms have been used to study atherosclerosis, each with varied success and limitations. ${ }^{3}$ Caobelli and Bengel highlight some exciting results from plaque imaging studies using SPECT, and the benefits of cadmium-zinc-telluride (CZT) SPECT over conventional SPECT with sodium iodide scintillation cameras.

The remit of our Review was specific to PET imaging; other noninvasive molecular imaging modalities used in atherosclerosis research include MRI with ultrasmall superparamagnetic iron oxide, ultrasonography with microbubble antibody ligands, multidetector CT with iodine-based or gold-based nanoparticles, and SPECT with ${ }^{99 \mathrm{~m}} \mathrm{Tc}$-labelling or ${ }^{111} \mathrm{In}$-labelling. Notably, in vivo leukocyte tracking using ${ }^{99 \mathrm{~m}} \mathrm{Tc}-\mathrm{labelled}$ autologous peripheral blood monocytes with serial SPECT imaging seems to correlate with vascular inflammation determined by ${ }^{18}$ F-FDG PET uptake, as well as disease severity assessed using MRI. ${ }^{49 \mathrm{~m} T c-l a b e l l e d}$ folate is another potentially useful imaging marker for plaque, which has been examined using ex vivo microSPECT imaging of human carotid arteries. ${ }^{5}$ At present, these molecular imaging techniques are experimental and, therefore, none is widely accessible for use in patients with atherosclerosis.

With any nuclear imaging method, whether SPECT or PET, inherent technical limitations of scanning equipment, reconstruction algorithms, and physical properties of the radioisotope influence imaging parameters including spatial resolution, which determine the capacity to image small structures. The latest-generation of ultrafast cardiac SPECT scanners equipped with CZT solid-state detectors and improved collimation systems seem to offer better image quality, with higher count sensitivity and superior spatial resolution than with conventional SPECT.6, ${ }^{6,7}$ For myocardial perfusion imaging, this new technology translates into substantial reductions in isotope dose and scan time, ${ }^{8,9}$ improved detection of partial thickness myocardial infarcts, ${ }^{10}$ and potentially increased sensitivity to detect multivessel disease. ${ }^{11}$ However, only limited evidence is currently available to support the use of SPECT for in vivo plaque imaging, and we are not aware of any prospective clinical study that has been conducted to evaluate a SPECT tracer for coronary plaque imaging. Furthermore, although conventional SPECT cameras are generally more commonplace in the clinical setting than PET, the same cannot be said for CZT SPECT, at least in the UK.

Technological upgrades and refinements will, undoubtedly, continue to advance frontiers in atherosclerosis imaging. Solid-state detectors, with avalanche photodiodes or digital silicon photomultipliers, have also been introduced in the latest PET scanners. ${ }^{12}$ These detectors provide improved spatial resolution and faster timing resolution, and are not sensitive to electromagnetic interference (which is of particular importance for hybrid PET-MRI). With ${ }^{82} \mathrm{Rb}$ or ${ }^{13} \mathrm{~N}$-ammonia cardiac PET, both electrocardiogram-gated myocardial perfusion imaging and dynamic quantitative assessment of coronary flow reserve can be reliably obtained, and combined with CT calcium scoring and/or high-resolution contrast angiography using the same PETCT scanner. ${ }^{13}$ Owing to the short half-life of
PET tracers used in perfusion imaging (76 s for ${ }^{82} \mathrm{Rb}$ and 9.9 min for ${ }^{13} \mathrm{~N}$-ammonia), ${ }^{14}$ a molecular marker of high-risk plaque activity, such as ${ }^{18} \mathrm{~F}-\mathrm{FDG}$ or ${ }^{18} \mathrm{~F}-\mathrm{NaF}$, can also feasibly be integrated; thus providing a complete anatomical, haemodynamic, and molecular assessment by capitalizing on state-of-theart hybrid cross-sectional imaging technology. This type of integrated approach might help to reduce the number of nondiagnostic scans and the need for repeat imaging, and streamline the selection of patients referred for invasive procedures.

Division of Cardiovascular Medicine, University of Cambridge, Box 110,

Addenbrooke's Centre for Clinical Investigation, Addenbrooke's Hospital, Hills Road,

Cambridge CB2 2QQ, UK (J.M.T., J.H.F.R.).

Correspondence to: J.H.F.R.

jhfr2@cam.ac.uk

Acknowledgements

J.M.T. is supported by a Wellcome Trust research training fellowship (104492/Z/14/Z) and the National Institute for Health Research (NIHR) Cambridge Biomedical Research Centre. J.H.F.R. is in part supported by the NIHR Cambridge Biomedical Research Centre, the British Heart Foundation, the Wellcome Trust, and the Academy of Medical Sciences.

Competing interests

The authors declare no competing interests.

1. Caobelli, F. \& Bengel, F. M. In vivo evaluation of atherosclerotic plaques and culprit lesions using noninvasive techniques. Nat. Rev. Cardiol. http://dx.doi.org/10.1038/nrcardio.2014 80-c1.

2. Tarkin, J. M., Joshi, F. R. \& Rudd, J. H. F. PET imaging of inflammation in atherosclerosis. Nat. Rev. Cardiol. 11, 443-457 (2014).

3. Joshi, F. R., Lindsay, A. C., Obaid, D. R., Falk, E. \& Rudd, J. H. F. Non-invasive imaging of atherosclerosis. Eur. Heart J. Cardiovasc. Imaging 13, 205-218 (2012).

4. van der Valk, F. M. et al. In vivo imaging of enhanced leukocyte accumulation in atherosclerotic lesions in humans. J. Am. Coll. Cardiol. 64, 1019-1029 (2014).

5. Jager, N. A. et al. Folate receptor- $\beta$ imaging using ${ }^{99 m} \mathrm{Tc}$-folate to explore distribution of polarized macrophage populations in human atherosclerotic plaque. J. Nucl. Med. http:// dx.doi.org/10.2967/jnumed.114.143180. 
6. Esteves, F. P. et al. Novel solid-state-detector dedicated cardiac camera for fast myocardial perfusion imaging: multicenter comparison with standard dual detector cameras. J. Nucl. Cardiol. 16, 927-934 (2009).

7. Takahashi, Y., Miyagawa, M., Nishiyama, Y., Ishimura, H. \& Mochizuki, T. Performance of a semiconductor SPECT system: comparison with a conventional Anger-type SPECT instrument. Ann. Nucl. Med. 27, 11-16 (2013).

8. Buechel, R. R. et al. Ultrafast nuclear myocardial perfusion imaging on a new gamma camera with semiconductor detector technique: first clinical validation. Eur. J. Nucl. Med. Mol. Imaging 37, 773-778 (2010).

9. Duvall, W. L. et al. Reduced isotope dose and imaging time with a high-efficiency CZT SPECT camera. J. Nucl. Cardiol. 18, 847-857 (2011).

10. Fukushima, Y. et al. Nuclear myocardial perfusion imaging with a cadmium-telluride semiconductor detector gamma camera in patients with acute myocardial infarction. Ann. Nucl. Med. 28, 646-655 (2014).

11. Gimelli, A. et al. Comparison between ultrafast and standard single-photon emission CT in patients with coronary artery disease: a pilot study. Circ. Cardiovasc. Imaging 4, 51-58 (2011).

12. Slomka, P. J., Berman, D. S. \& Germano, G New cardiac cameras: single-photon emission CT and PET. Semin. Nucl. Med. 44, 232-251 (2014).

13. Di Carli, M. F. et al. Clinical myocardial perfusion PET/CT. J. Nucl. Med. 48, 783-793 (2007).

14. Maddahi, J. \& Packard, R. R. S. Cardiac PET perfusion tracers: current status and future directions. Semin. Nucl. Med. 44, 333-343 (2014). 